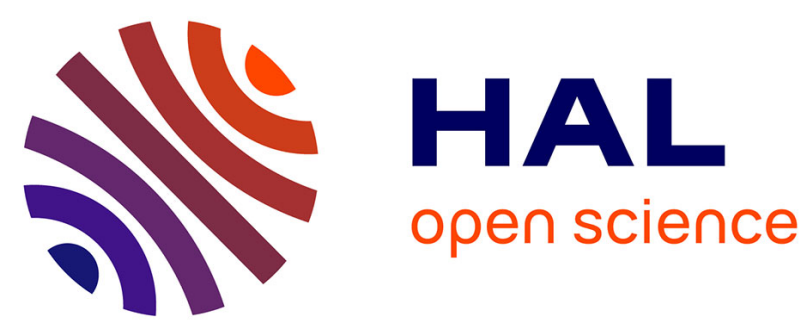

\title{
Oscillatory Light-Emitting Biopolymer Based Janus Microswimmers
}

\author{
Roberto María-hormigos, Alberto Escarpa, Bertrand Goudeau, Valerie \\ Ravaine, Adeline Perro, Alexander Kuhn
}

\section{- To cite this version:}

Roberto María-hormigos, Alberto Escarpa, Bertrand Goudeau, Valerie Ravaine, Adeline Perro, et al.. Oscillatory Light-Emitting Biopolymer Based Janus Microswimmers. Advanced Materials Interfaces, 2020, 7 (10), pp.1902094. 10.1002/admi.201902094 . hal-02510484

\section{HAL Id: hal-02510484 \\ https://hal.science/hal-02510484}

Submitted on 14 Sep 2020

HAL is a multi-disciplinary open access archive for the deposit and dissemination of scientific research documents, whether they are published or not. The documents may come from teaching and research institutions in France or abroad, or from public or private research centers.
L'archive ouverte pluridisciplinaire HAL, est destinée au dépôt et à la diffusion de documents scientifiques de niveau recherche, publiés ou non, émanant des établissements d'enseignement et de recherche français ou étrangers, des laboratoires publics ou privés. 


\section{Oscillatory Light-Emitting Biopolymer based Janus Microswimmers}

Roberto María-Hormigos, Alberto Escarpa, Bertrand Goudeau, Valérie Ravaine, Adeline Perro* and Alexander Kuhn*

\section{R. María-Hormigos, Prof. A. Escarpa}

Department of Analytical Chemistry, Physical Chemistry and Chemical Engineering, Chemical Research Institute "Andres M. Del Rio", Universidad de Alcalá, E-28871 Alcalá de Henares, Madrid, Spain.

Dr. A. Perro, Prof. V. Ravaine, Prof. A. Kuhn

Univ. Bordeaux, CNRS, Bordeaux INP, ISM UMR 5255, Site ENSCBP, 16 avenue Pey Berland, 33607 Pessac, France.

E-mail: adeline.perro@enscbp.fr; kuhn@enscbp.fr

Keywords: hydrogels, chemiluminescence, Janus micromotors, biopolymer, propulsion, Prussian Blue

Janus particles with a double functionality are synthesized by asymmetric loading of alginate hydrogel beads with Prussian Blue, leading to microswimmers with an original dynamic oscillatory behavior coupled to chemical light emission. This phenomenon results from the combination of two features: 1) Prussian Blue in the gel acts as a catalyst and enables concomitant light emission and oxygen production in the presence of luminol and hydrogen peroxide; 2 ) the hydrogel particle has a differential porosity distribution, leading to an asymmetric release of oxygen bubbles propelling the particle. The synthesis of these functional materials is achieved using an electric-field based symmetry breaking approach with ionically cross-linked alginate beads and can be applied to a wide range of particle sizes. Such light-emitting swimmers open up interesting perspectives for the elaboration of autonomous dynamic chemical systems. 


\section{Introduction}

Self-propelled artificial swimmers have been widely investigated during the last decade due to their great application potential in a biomedical,[1] environmental[2] and analytical context.[3] Motion of these micromotor systems is achieved by the conversion of an energy input, such as chemical fuels, magnetic fields, ultrasound waves, light or heat, into mechanical forces.[4] Special interest has been focused on micromotors powered by the decomposition of chemical fuel into gas bubbles, allowing to drive the swimmers autonomously without any external manipulation.[5] Regardless of the propulsion mechanism, controlling directional movement in certain environments is not straightforward, especially in an isotropic solution (e.g. without chemical or physical gradients) and more efforts should be focused on this aspect.[6] A simple way to address this challenge is restricting the propulsion mechanism to one dimension. Consequently, buoyancy forces experienced by particles and microsystems open up interesting avenues for propulsion concepts.[7]

Micromotor propulsion requires symmetry breaking and is commonly achieved by built-in structural synthesis. The synthesis of micromotor structures normally requires sophisticated processes and the use of surfaces (e.g. roll-up technology, physical vapor deposition) or membranes and specific experimental set-ups (e.g. electrodeposition, template based synthesis).[8] With this respect, bipolar electrochemistry also offers in a general sense an attractive alternative for the generation of asymmetric objects in a simple and straight forward way based on low cost equipment. The experimental set-up only needs two feeder electrodes and a solution in which the particles are suspended.[9] However, this method is mainly restricted to conductive materials like metal, carbon, conducting polymers and semiconductors.[9a, 10]

Hydrogel materials are particularly promising candidates for micromotor technology due to their packaging capabilities, cargo delivery and biocompatibility.[11] Their integration in microscale motors is desirable in order to meet the requirements for the development of advanced micromachines opening up new practical applications. In recent years, macroscopic soft hydrogel motors with self-propelling abilities were developed.[12] However, hydrogels are commonly nonconductive polymers preventing the use of bipolar electrochemistry. Nevertheless, efforts have been made to create Janus hydrogel beads by doping non-conductive natural hydrogels.[13] Additionally, alternative electrochemical processes, such as cation exchange through electrophoresis or electrolysis, were investigated to take advantage of the simplicity of electrochemistry set-ups even for non-conductive materials.[14]

In the present work, water electrolysis is applied to generate asymmetric non-conductive particles based on biocompatible hydrogels. Water splitting at the electrodes promotes a $\mathrm{pH}$ gradient in the electrochemical cell that induces an asymmetric modification of the porosity of hydrogel particles positioned between the electrodes. In this way Janus alginate beads loaded with Prussian Blue (PB) could be synthetized. The latter one acts as a catalyst to enable propulsion and simultaneous chemiluminescence. Indeed, in the presence of hydrogen peroxide, PB produces oxygen bubbles, which generate the propulsion. Due to their asymmetry, these beads present asymmetric gas entrapment-release profiles, which allow the generation of differential buoyancy forces and thus very efficient vertical motion. Simultaneously, PB catalyzes the oxidation of luminol by hydrogen peroxide, resulting in a concomitant chemiluminescence reaction, thus leading to light-emitting swimmers. The concept is validated at various scales by decreasing the particle size from the millimeter to the micrometer range, thereby demonstrating its robustness and tunability. 


\section{Results and Discussion}

\subsection{Millimeter sized Janus particle synthesis and characterization}

Typically, hydrogel beads are formed by the complexation of alginate polymer with $\mathrm{Ca} 2+$ cations. Other divalent $(\mathrm{Mg} 2+)$ or trivalent $(\mathrm{Fe} 3+)$ cations can also induce the gelation of alginate. In order to synthesize PB loaded beads, a sodium alginate solution is added drop-wise into a $0.05 \mathrm{M}$ iron (III) crosslinker solution. Spherical millimeter sized Fe3+-alginate beads are obtained after 15 min, due to the crosslinking induced by the iron ions. These particles are placed in a $\mathrm{K} 4[\mathrm{Fe}(\mathrm{CN}) 6]$ solution to obtain PB-alginate beads according to reaction 1.

$4 \mathrm{Fe} 3++3[\mathrm{Fell}(\mathrm{CN}) 6] 4-\rightarrow[4 \mathrm{FellI} 3[\mathrm{Fell}(\mathrm{CN}) 6]]$

Characteristic blue colored particles are obtained after the reaction. However, these particles crosslinked solely with iron (III) present a lack of stability, as they get dissolved rapidly after the reaction with ferrocyanide. In fact, due to the PB formation the ferric cations are no longer available for maintaining the hydrogel structure. Consequently, alginate gets dissolved, and the hydrogel particle disappears (Figure S1A).

In order to increase the stability of the PB-alginate particles, different gelation solutions are employed by mixing calcium (II) and iron (III) solutions, but keeping the total cation concentration at $0.05 \mathrm{M}$. Calcium cations act as inert stabilizer for the alginate hydrogel structure, since they do not react with ferrocyanide during the formation of PB. As can be seen in Figure S1, PB-alginate particles are more stable during the reaction with ferrocyanide when the molar ratio of iron (III) and calcium (II) is unity or lower. It is therefore necessary to find a compromise between introducing as many iron ions as possible, in order to generate a high loading with Prussian blue, but still keeping a reasonable amount of calcium ions to ensure sufficient stability. The best ratio that allowed generating enough Prussian blue and leading to stable particles was a 50/50 ratio of both ions in the initial solution. This means that we used a solution of $0.025 \mathrm{M} \mathrm{Fe} 3+$ mixed with $0.025 \mathrm{M}$ of $\mathrm{Ca} 2+$. However, it is not obvious that this ratio is also going to be the same in the final particle, because both cations might not have the same complexation kinetics and equilibrium association constants. Higher relative loadings with iron lead to unstable particles. All particles employed during this work were therefore synthesized with this equimolar proportion of iron (III) and calcium (II) cations in order to ensure on the one hand their stability during the subsequent experiments, but on the other hand to guarantee still a high enough content of PB for the catalytic reactions. These particles are named as Fe3+/Ca2+alginate.

In order to transform homogeneous particles into Janus objects, we have used water electrolysis at the electrodes of the electrochemical cell to create a pH-gradient.[14a] The experiment can be carried out following two different pathways, schematically described in Scheme 1. 


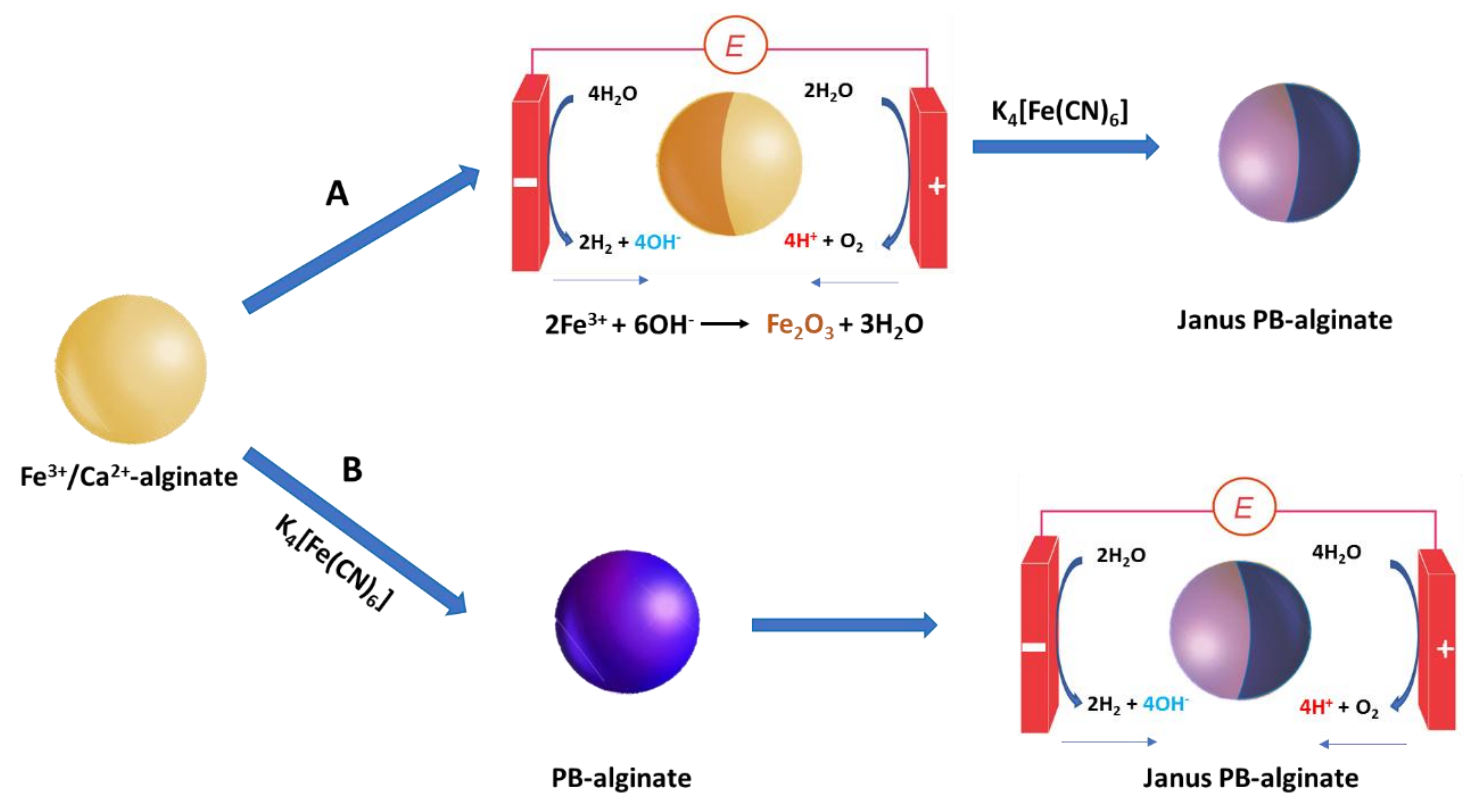

Scheme 1. Two different pathways for Janus PB-Alginate particle synthesis. Pathway A: Initial asymmetrization of $\mathrm{Fe} 3+/ \mathrm{Ca} 2+-$ alginate particles by electrochemical treatment. Water electrolysis produces $\mathrm{OH}$ - migration from the cathode towards the particle, leading to the modification of the alginate porosity and formation of $\mathrm{Fe} 2 \mathrm{O} 3$ on the exposed face. A subsequent reaction with ferrocyanide allows obtaining Janus PB-alginate particles. Pathway B: Homogeneous PB-alginate particles are obtained via the reaction of $\mathrm{Fe} 3+/ \mathrm{Ca} 2+-$ alginate particles with ferrocyanide and are then asymmetrically modified by an electrochemical treatment. The $\mathrm{pH}$ gradient leads to the partial PB elimination and changes the alginate porosity resulting in the formation of Janus PB-alginate particles.

For path $\mathrm{A}, \mathrm{Fe} 3+/ \mathrm{Ca} 2+-$ alginate beads were placed in an electrochemical cell, composed of two graphite electrodes, which induce water splitting reactions and the formation of hydrogen on the cathode and oxygen on the anode. As a result, hydroxyl ions and protons are generated at the cathode and anode, respectively. The migration of these ions produces a $\mathrm{pH}$ gradient in the electrochemical cell, exposing one face of the $\mathrm{Fe} 3+/ \mathrm{Ca} 2+-$ alginate bead to a basic medium and the other face to an acidic medium. A basic medium promotes the formation of species such as iron hydroxide and iron oxide. In a second step, a potassium ferrocyanide solution is then added to obtain the PB-alginate Janus particles. In pathway $\mathrm{B}$, the $\mathrm{Fe} 3+/ \mathrm{Ca} 2+$-alginate particles are first exposed to a potassium ferrocyanide solution in order to obtain isotropic PB-alginate beads. Subsequently, they are placed in the electrochemical cell and water electrolysis is performed in the same way as for path A.

Figure 1 shows the results observed for both options. For path A, the symmetry breaking is macroscopically observed via the formation of a dark-brown precipitate on the face exposed to basic media. Detailed steps and the visualization of the $\mathrm{pH}$ gradient with a $\mathrm{pH}$ indicator are presented in Figure S2A. SEM characterization of the surface morphology demonstrates that the micrometer scale the particle is asymmetric after the electrochemical treatment (Figure S3). The influence of the $\mathrm{pH}$ on the bead structure can be further examined by placing a symmetric Fe3+/Ca2+-alginate bead 
in basic or acid media. $0.01 \mathrm{M} \mathrm{NaOH}$ or $\mathrm{HNO} 3$ solutions were added to $\mathrm{Fe} 3+/ \mathrm{Ca} 2+$-alginate particles to simulate the local environment of these beads during the electrochemical treatment. As can be seen on the SEM images in Figure $\mathrm{S} 4, \mathrm{NaOH}$ produces an opening of micropores and channels on the surface of the particle, whereas acid medium does not produce any change in surface morphology.

Figure $1 \mathrm{C}$ shows the open pores formed on one side of the alginate polymer bead, which was in contact with the basic media. Figure 1D presents the other face, initially in contact with the acid environment, which remains smooth. These two different domains, coexisting in a single particle after the electrochemical treatment, confers the particles an asymmetric character.

For path $\mathrm{B}$, first a homogeneous distribution of $\mathrm{PB}$ inside the $\mathrm{Fe} 3+/ \mathrm{Ca2+-alginate}$ particles is observed (Figure 1B). Then, these particles are made asymmetric during the exposure to the $\mathrm{pH}$ gradient generated by the electrochemical approach. Janus particles presenting a macroscopic asymmetry with a blue and light-yellow face are obtained. Under the influence of the $\mathrm{pH}$ gradient formed in the electrochemical cell, PB is removed at the face exposed to the basic medium (Figure S2B). Analog to what has been observed for pathway $A$, microscopy reveals that the surface of the bead presents two distinct porosity domains.
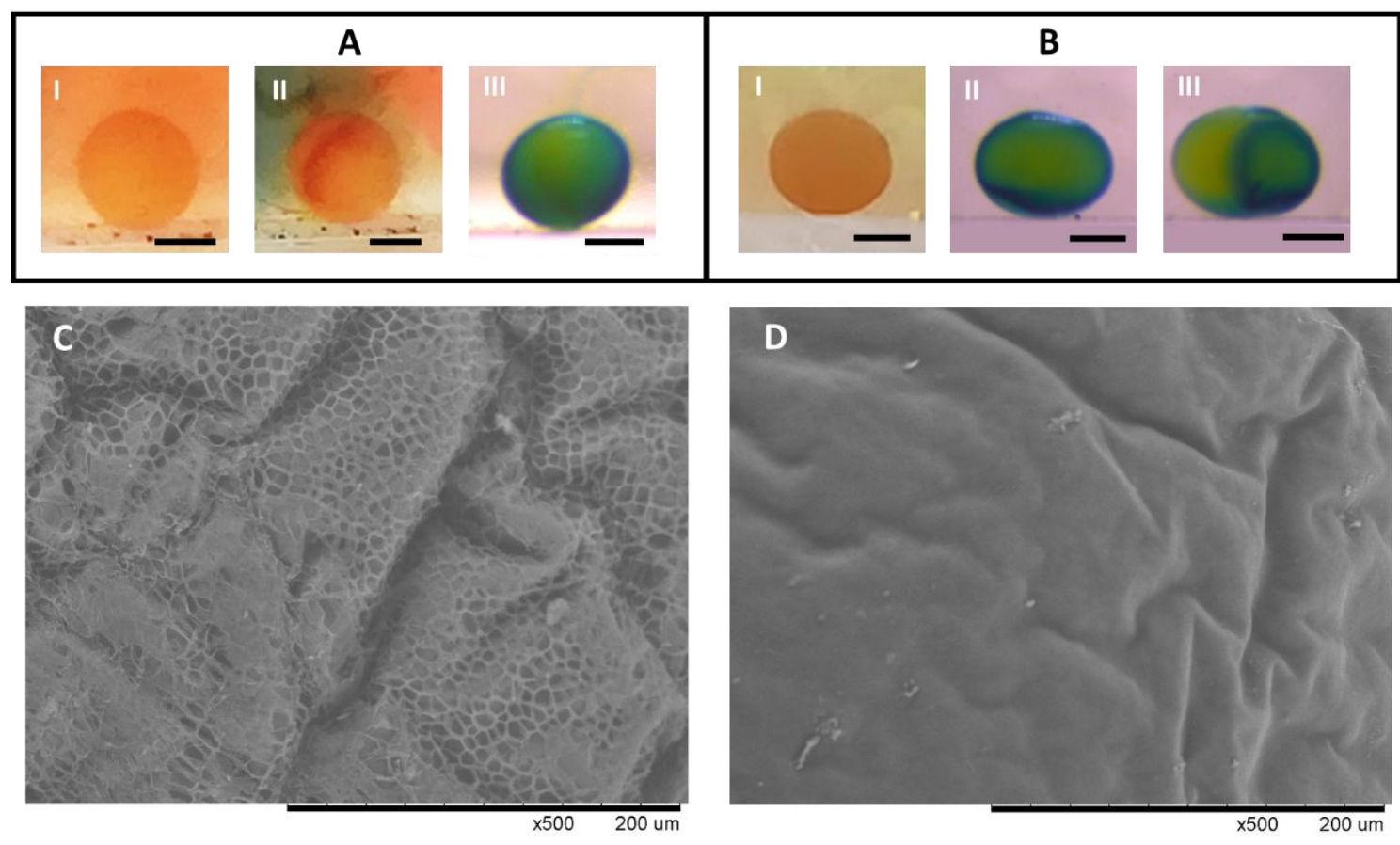

Figure 1. Images of millimetric particles during the synthesis of Janus PB-alginate particles using the two pathways. (A) I) Symmetric Fe3+/Ca2+-alginate particle, II) after the electrochemical treatment, III) Janus particle loaded with Prussian Blue. (B) I) Symmetric Fe3+/Ca2+-alginate particle, II) Symmetric Prussian Blue loaded Fe3+/Ca2+-alginate particle, III) Janus particle obtained after the electrochemical treatment. Scale bars $1 \mathrm{~mm}$. (C) SEM image of the Fe3+/Ca2+-alginate particle face which was exposed to basic $\mathrm{pH}$ in the electrochemical cell $(\mathrm{pH}>9)$. (D) SEM image of the Fe3+/Ca2+alginate particle face which was exposed to acid $\mathrm{pH}$ in the electrochemical cell $(\mathrm{pH}<4)$. 
In terms of final performance, there is no significant difference between both synthesis pathways. During pathway $B$ the generation of asymmetry is better visible during the experiment due to the dark blue color of the initially homogeneous particle.

\subsection{Motion and chemiluminescence of millimeter sized Janus particles}

The soft structure of hydrogels allows for loading and release of cargo molecules.[11] In this work, we explore the capacity of biobased polymer beads to produce, store and release gas, leading to a modulation of the particle buoyancy. This phenomenon can be used to trigger an oscillatory vertical motion at the centimeter scale. Scheme 2 illustrates this concept of periodic buoyancy shift. PBloaded Janus particles catalyze $\mathrm{H} 2 \mathrm{O} 2$ decomposition. This forms $\mathrm{O} 2$ bubbles, which diffuse out of the particle. The overall dynamics of the particle depends in a subtle way on its porosity. Three different experimental situations can be encountered. In the first case, the PB-alginate particles, before any electrochemical treatment, present a smooth and non-porous surface, which hinders bubble release from the particle (Scheme $2 \mathrm{~A}$ ). As a result, more and more gas gets trapped inside the bead and the overall buoyancy increases, which pushes the bead towards the water/air interface, where it stays permanently.

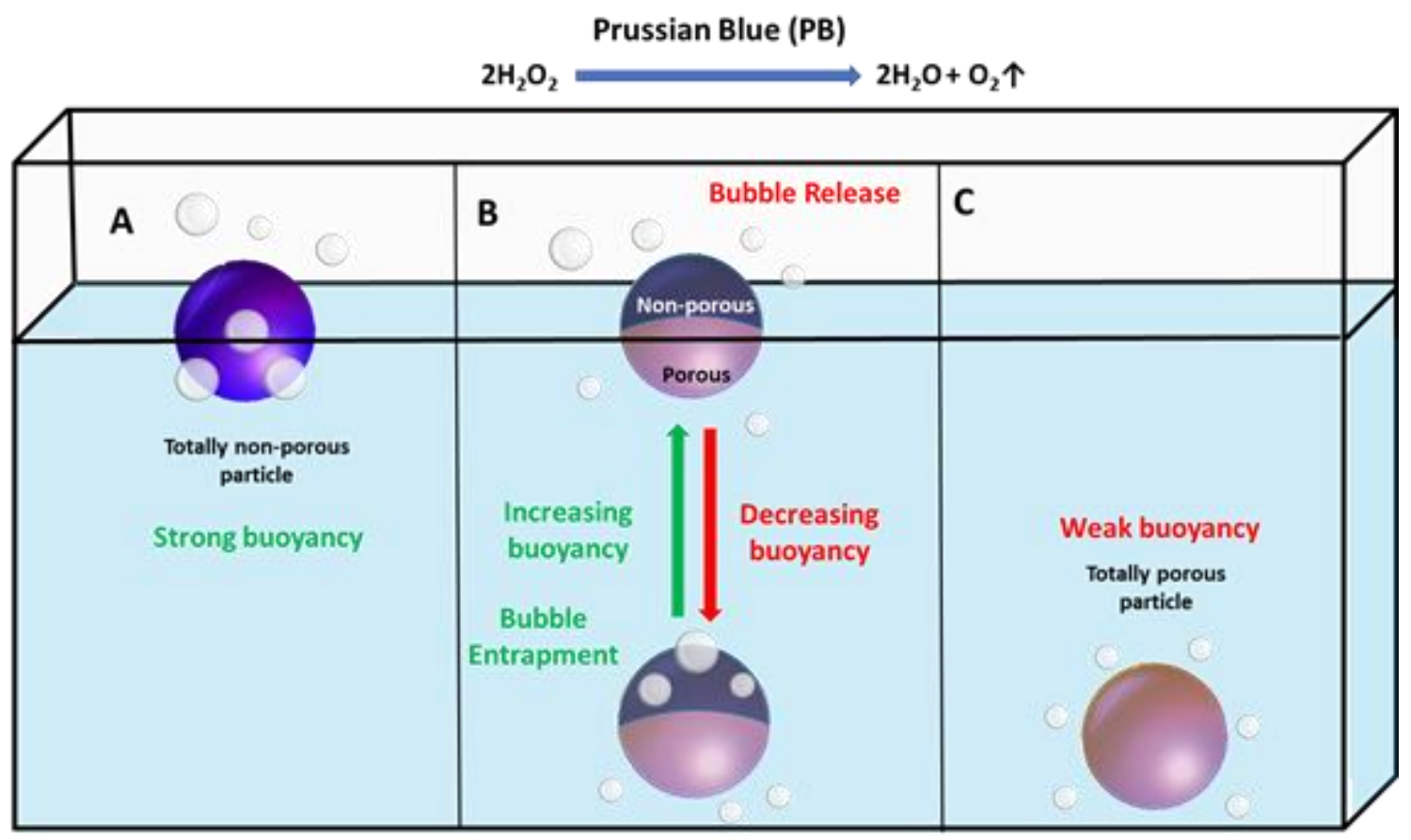

Scheme 2. Schematic illustration of the different dynamic behaviour of PB-alginate particles. (A) For isotropic non-porous particles, $\mathrm{O} 2$ produced by the catalytic action of $\mathrm{PB}$ is trapped, leading to stationnary floating. (B) For Janus particles, $\mathrm{O} 2$ gas is trapped in the non-porous part of the particles, increasing their overall buoyancy, which leads to ascending motion. The gas is released when reaching the air-water interface, inducing a sinking of the particles. (C) For homogeneous particles, presenting a high porosity due to preliminary treatment with $\mathrm{NaOH}$, gas bubbles are released constantly and isotropically from the pores, leading to a particle without motion. 
In the second case, the Janus PB-alginate particles present an asymmetry in surface porosity (Scheme 2B). Gas can easily escape from one side of the particle thanks to the large pores. On the other side, which does not present any porosity, oxygen bubbles get trapped, causing an overall increase of the particle buoyancy, leading to an ascending motion. When the particle finally reaches the water-air interface, the bubbles trapped in the upper part get released, leading to a descending motion. This cycle is repeated when the particle is again diving in the bulk solution, creating an oscillatory vertical motion. In the third scenario, totally porous PB-alginate particles were obtained by opening channels after their immersion in basic media (Scheme $2 \mathrm{C}$ ). When hydrogen peroxide is added to these porous beads, the generated gas bubbles are constantly released, thus preventing an ascending movement due to the weak buoyancy of these particles.

Figure 2 shows time-laps images taken from Video S1 and Video S2 corresponding to the behavior of Janus and symmetric PB-alginate particles in a solution containing $10 \%$ of $\mathrm{H} 2 \mathrm{O} 2$. One can distinguish the three different situations $(A, B, C)$, demonstrating that oscillatory vertical motion is only observed for Janus particles.

A

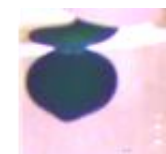

$3.5 \mathrm{~s}$

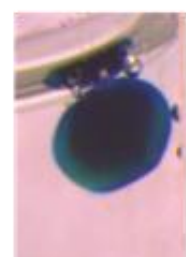

B

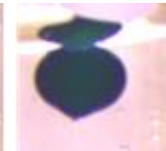

$8.6 \mathrm{~s}$

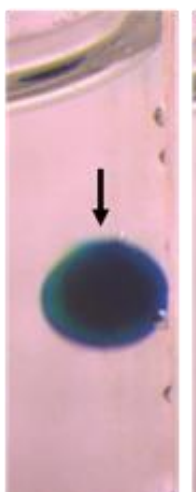

$6.8 \mathrm{~s}$

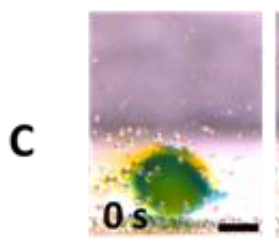

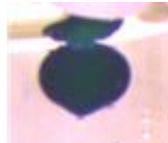

$11.7 \mathrm{~s}$

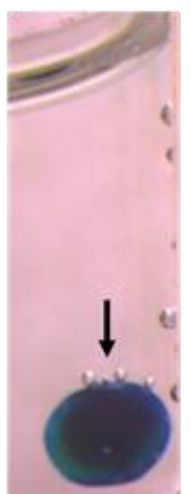

$11.3 \mathrm{~s}$

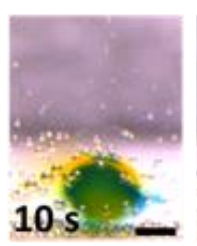

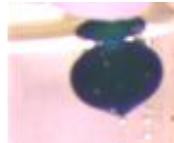

$14.7 \mathrm{~s}$

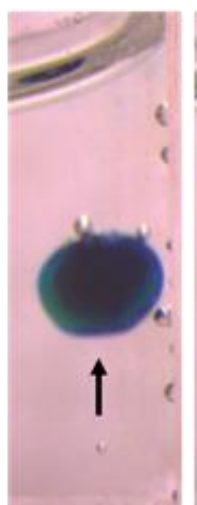

$12.5 \mathrm{~s}$

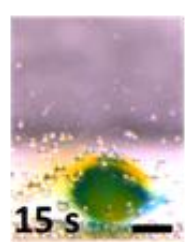

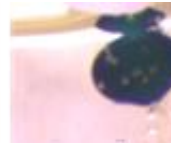

$18.5 \mathrm{~s}$
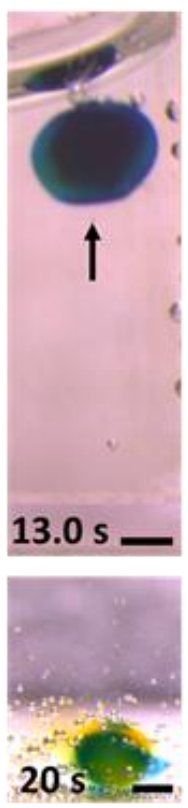

$23.8 \mathrm{~s}$
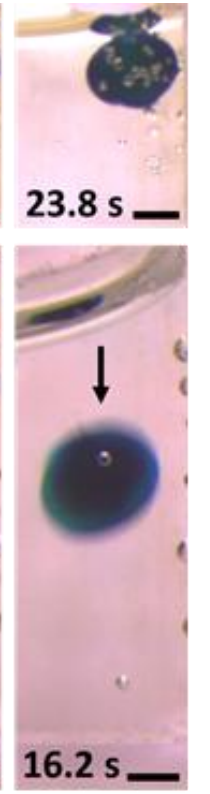

$16.2 \mathrm{~s}$

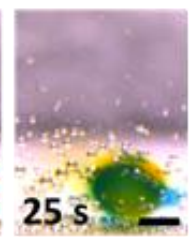

Figure 2. (A) Time-laps images of a symmetric PB-alginate particle with a totally non-porous surface. (B) Time-laps images of an asymmetric Janus PB-alginate particle taken from Video S1 (see SI). (C) Time-laps images of a particle with a homogeneous porosity after treatment with $\mathrm{NaOH}$ to open pores and channels. Images taken from Video S2 (see SI). Conditions: $10 \% \mathrm{H} 2 \mathrm{O} 2$. Scale bars $1 \mathrm{~mm}$. 
Periodic motion is achieved for both Janus particle preparation pathways employed in this work. Consequently, the key parameter, which controls the buoyancy effect, is linked to the microscopic structure. The bead porosity is the same for both pathways, the essential ingredient being the presence of one porous face and another with a smooth morphology. Janus PB-alginate millimeter sized particles move rather slowly, due to the size related inertia. An average speed of $1.5 \pm 0.7$ $\mathrm{mm} / \mathrm{s}$ ( $\sim 1$ bodylength $/ \mathrm{s})$ is recorded during ascension and they are sinking with $4.4 \pm 1.1 \mathrm{~mm} / \mathrm{s}$ ( 2 bodylengths/s). More precisely, the obtained particles move with $1.9 \pm 0.5 \mathrm{~mm} / \mathrm{s}$ (pathway A) and $1.1 \pm 0.3 \mathrm{~mm} / \mathrm{s}$ (pathway B) in the ascending motion, and $4.3 \pm 1.2 \mathrm{~mm} / \mathrm{s}$ (pathway A) and $4.5 \pm 1.1$ $\mathrm{mm} / \mathrm{s}$ (pathway $B$ ) in the descending motion. A very similar speed is obtained for both approaches, thus illustrating the independence of the dynamic performance of the Janus beads with respect to the synthesis route.

The motion of these beads can be easily tracked using the catalytic properties of the incorporated PB for the chemiluminescent reaction of luminol and $\mathrm{H} 2 \mathrm{O} 2$ in basic media. Figure $3 \mathrm{~A}$ illustrates the chemiluminescence, transforming the Janus PB-alginate particles into light-emitting swimmers. Blue light $(\lambda=425 \mathrm{~nm})$ is emitted by the particle, allowing tracking the particles in the solution by their light emission.

A

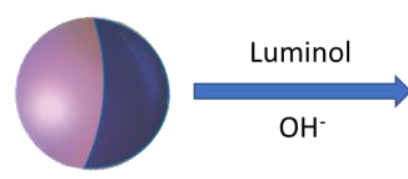

B
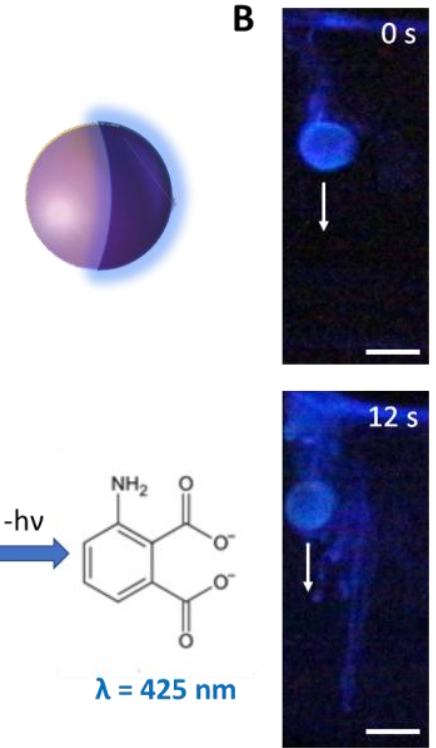
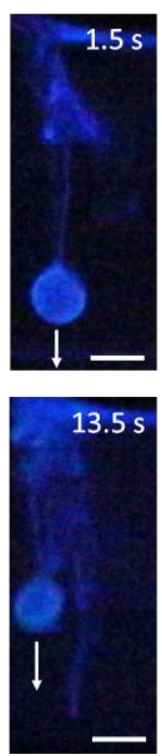
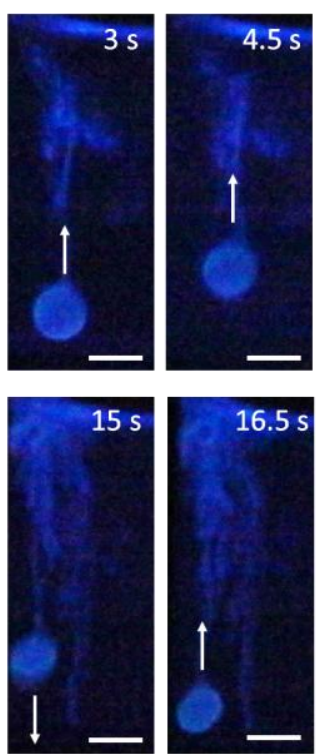

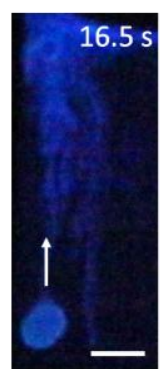

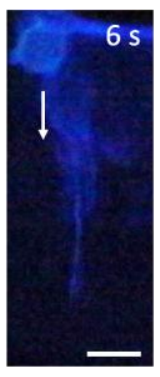

$18 \mathrm{~s}$

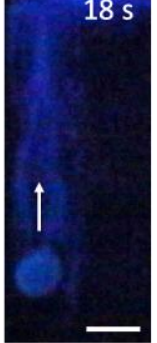

Figure 3. (A) Schematic mechanism of chemiluminescence due to the reaction of luminol with $\mathrm{H} 2 \mathrm{O} 2$, mediated by the Prussian Blue-alginate particles in basic media. (B) Time-laps chemiluminescence images of the Janus PB-alginate particle, undergoing an oscillatory motion. Conditions: $0.5 \mathrm{~s}$ time of exposure, $0.01 \mathrm{M}$ Luminol, $0.1 \mathrm{M} \mathrm{NaOH}, 10 \% \mathrm{w} / \mathrm{v} \mathrm{H} 2 \mathrm{O} 2$. Scale bars $2 \mathrm{~mm}$.

The Janus character of the particle leads to an asymmetric light emission as can be seen in Figure $\mathrm{S} 5$. Figure 3B shows images of a Janus PB-alginate particle moving periodically up and down in the solution accompanied by light emission. Chemiluminescence allows an easy tracking of the motion, which can be very useful in the context of autonomous swimmers. This concept can be applied to other types of movement and swimmers, as well as at a smaller scale. 


\subsection{Micrometer particles}

In order to enhance the mobility of the particles, miniaturized Fe3+/Ca2+-alginate spheres were synthesized by a microfluidic approach, in which a water-in-oil drop method is employed to obtain spherical particles between 125 and $500 \mu \mathrm{m}$ (see experimental section). Janus PB-alginate particles were obtained by following pathway $\mathrm{B}$, previously described for millimeter sized particles. The $\mathrm{pH}$ induced asymmetrization in the electrochemical cell is illustrated in Figure 4.

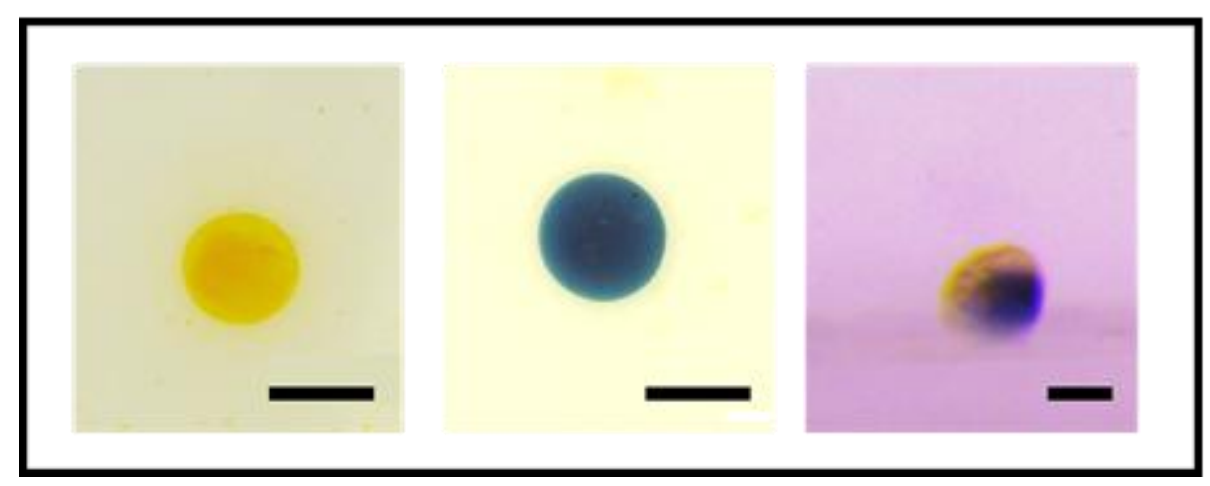

Figure 4. Images of a microparticle at different steps of the synthesis of Janus PB-alginate particles. On the left is shown a homogeneous $\mathrm{Fe} 3+/ \mathrm{Ca} 2+-$ alginate particle. The center image illustrates a PBalginate particle after the reaction with $\mathrm{K} 4[\mathrm{Fe}(\mathrm{CN}) 6]$. On the right side is a Janus $\mathrm{PB}$-alginate particle after the $\mathrm{OH}$ - front has reached the particle, leading to $\mathrm{PB}$ removal from one face of the particle.

Scale bars $250 \mu \mathrm{m}$.

First, spherical yellow Fe3+/Ca2+-alginate particles were synthesized by the microfluidic approach. Then, ferrocyanide was incorporated in order to obtain homogeneous PB-alginate beads. Finally, the particles were exposed to the $\mathrm{pH}$ gradient, established in the electrochemical cell by water electrolysis. The face exposed to the basic environment undergoes PB degradation, turning the color to yellow. Figure 5A shows a Janus PB-alginate microparticle, emitting blue light, characteristic for luminol chemiluminescence. This blue light emission can be used for particle tracking and for analytical purposes. However, the time required to collect sufficient light is conditioned by the performance of the camera and takes under the current conditions around 30s, which makes it difficult to obtain a clear record of the light emitted by a moving particle. 

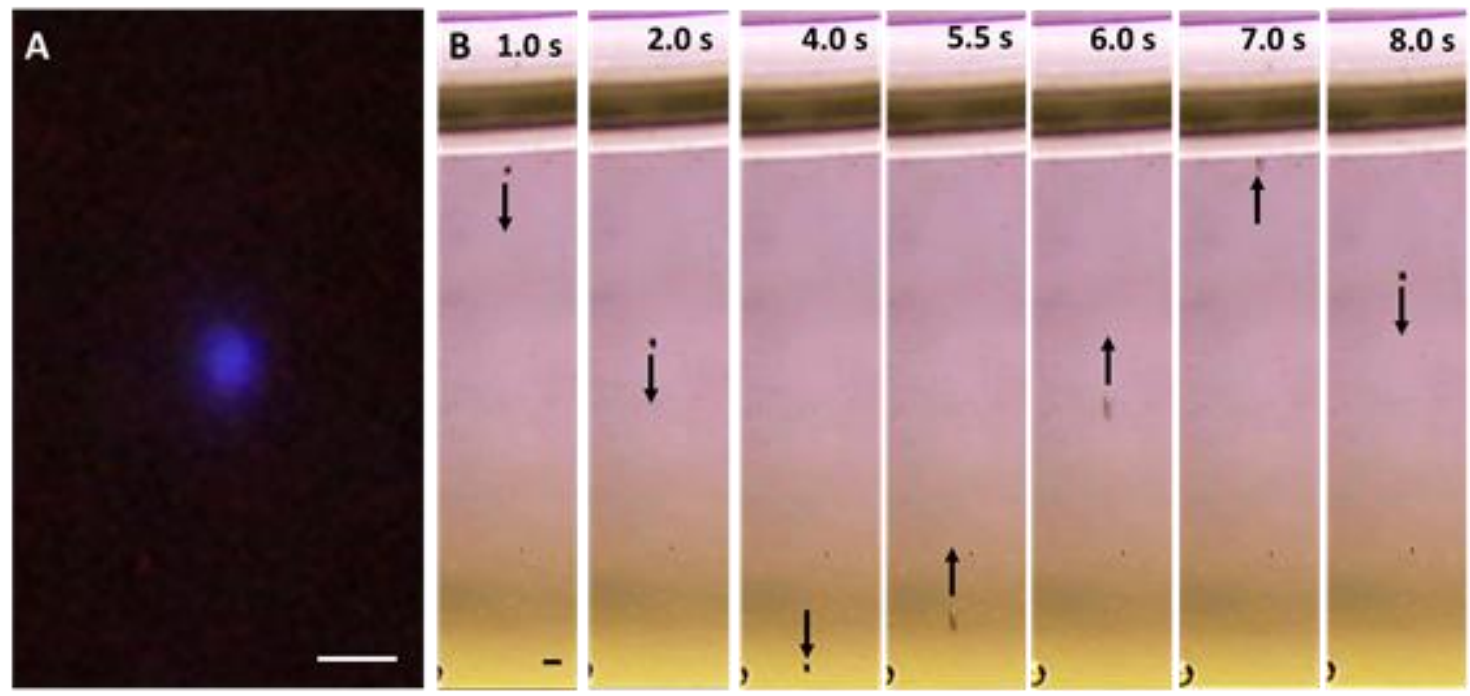

Figure 5. (A) Image of blue light chemiluminescence of a Janus PB-alginate particle. Conditions: $30 \mathrm{~s}$ camera exposure, $0.01 \mathrm{M}$ Luminol, $0.1 \mathrm{M} \mathrm{NaOH}, 1 \% \mathrm{w} / \mathrm{v} \mathrm{H} 2 \mathrm{O} 2$. (B) Time-laps images of a Janus PBalginate microparticle with $3 \% \mathrm{H} 2 \mathrm{O} 2$ taken from Video S3. Scale bars $250 \mu \mathrm{m}$.

Figure 5B shows the motion of a Janus PB-alginate microparticle in 3\% $\mathrm{H} 2 \mathrm{O} 2$ (frames are taken from Video S3). The particle moves along an oscillatory vertical trajectory with a mean speed of $3.2 \pm 0.1$ $\mathrm{mm} / \mathrm{s}$ ( 25 bodylengths $/ \mathrm{s}$ ) while rising, and with a mean speed of $0.94 \pm 0.01 \mathrm{~mm} / \mathrm{s}$ (7.5 bodylengths/s) when sinking. This is a substantial increase in speed compared to millimeter-sized particles, which can be attributed to a smaller inertia.

Figure $\mathrm{S} 6$ shows the behavior (in terms of speed profile and position) of the particle during its movement. A detailed study allows correlating the observed dynamics with previous work that uses this mechanism. The forces involved in such a process were described by Inagi's group with equations 1 and 2: 15

Descending: FNet $=$ FGravity - FBuoyancy - FDrag

(Equation 1)

Ascending: FNet $=$ FBuoyancy - FGravity - FDrag (Equation 2)

When PB Janus particles are immersed in fuel solution, the decomposition of $\mathrm{H} 2 \mathrm{O} 2$ produces a large amount of oxygen bubbles, which generate an extra buoyancy force. At the beginning (step 1) the particle resides at the air/water interface $(F N e t=0)($ Figure S6A). Next, the bubbles are released, and due to FGravity (FNet $>0$ ) the particle experiences a sudden speed increase, followed by a slowingdown induced by the continuous bubble generation (Figure S6 B). Then, the particle speed reaches zero, which translates an equilibrium between the different forces (Figure $\mathrm{S6C}$ ). Due to the continuous formation of bubbles, the Janus particle moves again upwards in the fuel solution (FBuoyancy > FGravity+FDrag), accompanied by an increase in speed (Figure S6D). Finally, the particle reaches once more the air/water interface and the cycle starts again.

The forces involved in buoyancy propulsion are defined by equations 3 to 5 :

FGravity $=m$ Particle $g=\rho$ Particle $g$ VParticle $=\rho$ Particle $g(4 / 3 \pi \gamma$ Particle3) $\quad$ (Equation 3$)$

FBuoyancy $=$ FBubble buoyancy + FParticle buoyancy (Equation 4)

FDrag $=6 \pi \eta \gamma$ Particle $u \quad$ (Equation 5) 
where $\mathrm{mParticle}$ is the particle mass, $\mathrm{g}$ is the constant gravity acceleration, $\rho$ Particle is the particle density, VParticle is the particle volume, $\gamma$ Particle is the particle radius, FBubble buoyancy is the bubble's contribution to buoyancy force, FParticle buoyancy is the particle's constant contribution to buoyancy force, $\eta$ is the medium viscosity, $\gamma$ Particle is the particle radius and $u$ the particle speed.

FGravity is a constant force in the system, which depends on the cube of the particle radius (Equation 3). Consequently, this force is about 500 times higher for millimeter sized particles $(\gamma=1 \mathrm{~mm})$ compared to micrometer particles $(\gamma=0.125 \mathrm{~mm})$.

FDrag is a force that is opposite to particle displacement, and directly proportional to its size. (equation 5).

Consequently, the ascending motion of the particles is governed by the buoyancy forces from the particles and the bubbles, which are defined by equation 6 and 7 :

FParticle buoyancy $=\rho$ Solution g VParticle $=\rho$ Solution $g(4 / 3 \pi(\gamma$ Particle $) 3) \quad$ (Equation 6$)$

FBubble buoyancy $=\rho$ Solution $g \Sigma$ VBubbles $=\rho$ Solution $g(4 / 3 \pi \Sigma(\gamma$ Bubbles $) 3)$ (Equation 7)

As can be seen from equation 6, particles have a constant buoyancy force, which depends, as FGravity, on the volume of the particle. However, this force is smaller than FGravity, thus the necessity of having oxygen bubbles to lift up the particles. FBubble buoyancy is therefore the main force involved in the oscillatory propulsion. At the same time in equation 7, FBubble buoyancy is a function of the number and size of oxygen bubbles. For millimeter size particles ( $\gamma=0.125 \mathrm{~mm})$, a dozen of oxygen bubbles are formed on the particle surface due to the low curvature radius. Therefore, these bubbles induce a fast ascending motion. The residence time at the air/water interface, which correspond to the release of the oxygen bubbles is on the order of a few seconds. For micrometer particles, due to the high curvature radius, only one bubble contributes to the ascending motion, which induces a decrease of the speed (2-fold). As the particle reaches the interface, the release of just one bubble decreases the residence time ( $1 \mathrm{~s})$. Consequently, the characteristic cycle time is a function of the bubble formation and release, which depends on the diameter of the particles.

\section{Conclusion}

In conclusion, we have successfully synthesized Janus Prussian Blue alginate beads using a pH gradient generated by water electrolysis in an electrochemical cell. Under the influence of this gradient, it was possible to generate different porosities at the two faces of the particles. These asymmetric particles show oscillatory motion due to periodic changes in the buoyancy forces resulting from the decomposition of hydrogen peroxide catalyzed by PB. Moreover, these particles simultaneously emit light generated by the PB catalyzed oxidation of luminol. The synthesis can be adapted to various size scales and allows changing structural parameters, including the size of the pores and the degree of asymmetry, in order to tune the particle speed. Buoyancy effects might be further integrated into a wider range of micromotor systems, allowing opening up new opportunities to fine-tune swimmer motion in three dimensions. Moreover, the PB loading can be extended to other chemiluminescent molecules in order adapt the system to applications in analytical chemistry. We anticipate that in the long run such particles can be used for different other applications 
including active mixing, active transport i.e. of biomolecules adsorbed at the gas/liquid interface and for controlled release.

\section{Experimental Section}

Reagents and Equipment. Sodium alginate (C6H9NaO7, Sigma), iron(III) chloride (FeCl3.xH2O, Sigma), calcium chloride ( $\mathrm{CaCl} 2,>93 \%$, Sigma), potassium iron(II) hexacyanoferrate (K4Fe(CN)6.3H2O, Fluka), hydrogen peroxide ( $\mathrm{H} 2 \mathrm{O} 2,27 \%$, Sigma), luminol (C8H7N3O2, $97 \%$, Sigma), sodium hydroxide ( $\mathrm{NaOH},>97 \%$, Fluka), nitric acid (HNO3, $70 \%$, Sigma), sodium dodecyl sulfate (CH3(CH2)110SO3Na, $>99 \%$, Sigma), hexane $\left(\mathrm{CH} 3(\mathrm{CH} 2) 4 \mathrm{CH} 3\right.$, anhydrous $95 \%$, Sigma), Span $80\left(\mathrm{C}_{24} \mathrm{H}_{44} \mathrm{O}_{6}\right.$, Sigma) and pHindicator solution pH $4.0-10.0$ (Supelco) were used as received without further purification. All aqueous solutions were prepared using Milli-Q water (Millipore, resistivity $18.2 \mathrm{M} \Omega \cdot \mathrm{cm}$ at $25^{\circ} \mathrm{C}$ ).

The microfluidic setup was made of glass capillaries, with inner diameters of $75 \mu \mathrm{m}$ and $250 \mu \mathrm{m}$ from Polymicro technologies for alginate-water solution and oil, respectively. The surface of the outer capillary was modified in order to increase its hydrophobicity.[16] T-junction and tubing sleeves from UpChurch Scientific were used to generate aqueous droplets. Fluids were pumped by two independent single-syringe pumps (SP100iZ, World Precision Instruments). Scanning electron microscopy was carried out on a TM-1000 tabletop microscope (Hitachi) to characterize hydrogel particles. A thin gold layer was sputtered on the particles before their characterization. Bright field images and videos were recorded using a planar CCD camera combined with LED based background lightning. Chemiluminescence images were recorded by a classic camera (Canon EOS 70D, Canon).

Alginate particle synthesis. Millimeter sized alginate hydrogel spheres were prepared in one step by the dropwise addition of $10 \mu \mathrm{L}$ sodium alginate solution $(2.0 \mathrm{wt} \%)$ into a gelation bath containing different proportions of iron(III) chloride and calcium chloride. The droplets of sodium alginate solidify into gel particles in the cation containing solution. After 15 minutes, the beads were solid enough for manipulation due to the crosslinking induced by the iron and calcium ions.[18] Subsequently, the hydrogel beads were cleaned three times with water and stored in ultrapure water. The particles remain stable without changes in their morphology for 3 days.

Micrometer alginate hydrogel spheres were prepared by a co-flow microfluidic process. A sodium alginate solution containing $5 \mu \mathrm{M}$ of SDS as surfactant was pumped into a glass capillary (inner diameter $75 \mu \mathrm{m}$, flow rate of $0.5 \mathrm{~mL} / \mathrm{h}$ ). Then, a second flow of hexane containing $0.01 \mathrm{wt} \%$ of Span 80 as surfactant was injected into a glass capillary (inner diameter $250 \mu \mathrm{m}$, flow rate $3 \mathrm{~mL} / \mathrm{h}$ ) via a Tjunction to create water-in-oil droplets. The droplets were collected in a gelation bath containing iron(III) chloride $0.025 \mathrm{M}$, calcium chloride $0.025 \mathrm{M}$ and SDS $5 \mu \mathrm{M}$. These beads (125-500 $\mu \mathrm{m}$ ) were kept in the gelation bath for 2 hours. Subsequently the hydrogel spheres were washed by centrifugation at $1000 \mathrm{rpm}$ during 4 minutes, two times with acetone to remove hexane and Span 80 residues and three times with ultrapure water. The particles were used the same day to ensure a stable shape.

Prussian Blue-alginate (PB-Alginate) Janus particle modification. PB-alginate Janus particles were synthesized by two different pathways. In the first one the Fe3+/Ca2+-alginate particles were asymmetrically modified at room temperature, in a quartz UV-Visible cuvette, used as an electrochemical cell. The alginate beads were positioned between two graphite electrodes (working distance of $0.5 \mathrm{~cm}$ ). The cell was filled with $5 \mathrm{mM}$ NaNO3 solution to ensure the current flow. A potential of $15 \mathrm{~V}$ was applied to promote water splitting at the two feeder electrodes during 5 minutes. Then, $0.1 \mathrm{mM}$ of $\mathrm{K} 4[\mathrm{Fe}(\mathrm{CN}) 6)]$ was added to obtain PB-alginate Janus particles. 


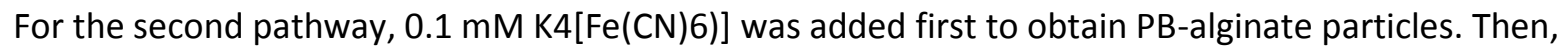
these particles were placed in the electrochemical cell, and a potential of $15 \mathrm{~V}$ was applied for 5 minutes.

Recording light-emitting particles. PB-alginate Janus particles were used as catalyst for the chemiluminescence reaction of luminol and $\mathrm{H} 2 \mathrm{O} 2$. PB-alginate Janus particles were put into a $2 \mathrm{~mL}$ quartz UV-Visible cell in the presence of $\mathrm{H} 2 \mathrm{O} 2$ solutions for millimeter sized particles and microparticles, respectively. After several minutes, while the particles are moving, $1 \mathrm{~mL}$ solution of luminol in $\mathrm{NaOH}$ was added. $10 \% \mathrm{H} 2 \mathrm{O} 2,0.01 \mathrm{M}$ luminol and $0.1 \mathrm{M} \mathrm{NaOH}$ were employed as final concentrations for millimeter sized particles $(2 \mathrm{~mm})$ and $3 \% \mathrm{H} 2 \mathrm{O} 2,0.01 \mathrm{M}$ luminol and $0.1 \mathrm{M} \mathrm{NaOH}$ were employed for micrometer particles $(250 \mu \mathrm{m})$. All chemiluminescence experiments were performed in the absence of light.

Supporting Information

Supporting Information is available from the Wiley Online Library or from the author.

\section{Acknowledgements}

R. Maria-Hormigos acknowledges the FPI fellowship from the Spanish Ministry of Economy and Competitiveness (BES-2015-072346). The authors thank Chengyu Li, Heimana Harua for preliminary experiments and Bhavana Gupta for optical recording. The project has also been partially funded by the European Research Council (ERC) under the European Union's Horizon 2020 research and innovation program (grant agreement no741251, ERC Advanced grant ELECTRA).

\section{References}

[1] a) W. Gao, J. Wang, Nanoscale 2014, 6, 10486; b) M. Guix, C. C. Mayorga-Martinez, A. Merkoçi, Chem. Rev. 2014, 114, 6285; c) J. Wang, Nanomachines: Fundamentals and Applications, Wiley-VCH, Weinheim, Germany 2013.

[2] a) L. Soler, V. Magdanz, V. M. Fomin, S. Sánchez, O. G. Schmidt, ACS Nano. 2013, 7, 9611; b) L. Soler, S. Sánchez, Nanoscale 2014, 6, 7125; c) W. Z. Teo, R. Zboril, I. Medrik, M. Pumera, Chem. Eur. J. 2016, 22, 4789; d) B. Jurado-Sánchez, J. Wang, Environ. Sci.: Nano. 2018, 5, 1530;

[3] a) B. Jurado-Sánchez, A. Escarpa, TrAC. 2016, 84, 48; b) W. Duan, W. Wang, S. Das, V. Yadav, T. E. Mallouk, A. Sen, Annu. Rev. Anal. Chem. 2015, 8, 311; c) B. Jurado-Sánchez. A. Escarpa, Electroanal. 2017, 29, 14;

[4] a) W. Gao, A. Pei, R. Dong, J. Wang, J. Am. Chem. Soc. 2014, 136, 2276; b) H. Wang, G. J. S. Moo, M. Pumera, ACS Nano. 2016, 10, 5041; c) M. Guix, A. K. Meyer, B. Koch, O. G. Schmidt, Sci. Rep. 2016, 6, 21701; d) G. Loget, A. Kuhn, Nat. Commun. 2011, 2, 535; e) J. Li, T. Li, T. Xu, M. Kiristi, W. Liu, Z. Wu, J. Wang, Nano Lett. 2015, 15, 4814; f) W. Wang, S. Li, L. Mair, S. Ahmed, T. J. Huang, T. E. Mallouk, Angew. Chem. Int. Ed. 2014, 53, 3201; g) R. Q. Rudy, G. L. Smith, D. L. DeVoe, R. G. Polcawich, J. Microelectromech. Syst. 2015, 24, 108; h) L. Soler, C. Martínez-Cisneros, A. Swiersy, S. Sánchez, O. G. Schmidt, Lab Chip 2013, 13, 4299; i) M. Xuan, Z. Wu, J. Shao, L. Dai, T. Si, Q. He, J. Am. Chem. Soc. 2016, 138, 6492; j) H. Eskandarloo, A. Kierulf, A. Abbaspourrad, Nanoscale 2017, 9, 12218; k) R. Dong, Y. Hu, Y. Wu, W. Gao, B. Ren, Q. Wang, Y. Cai, J. Am. Chem. Soc. 2017, 139, 1722; 
[5] a) W. Gao, M. D'Agostino, V. Garcia-Gradilla, J. Orozco, J. Wang, Small 2013, 9, 467; b) J. Orozco, L. A. Mercante, R. Pol, A. Merkoçy, J. Mater. Chem. A. 2016, 4, 3371; c) B. Jurado-Sánchez, M. Pacheco, J. Rojo, A. Escarpa, Angew. Chem. Int. Ed. 2017, 56, 6957; d) J. A. M. Delezuk, D. E. RamírezHerrera, B. Esteban-Fernández de Ávila, J. Wang, Nanoscale 2017, 9, 2195;

[6] a) T. R. Kline, W. F. Paxton, T. E. Mallouk, A. Sen, Angew. Chem. Int. Ed., 2005, 44, 744; b) W. F. Paxton, A. Sen, T. E. Mallouk, Chem. Eur. J., 2005, 11, 6462. c) W. Wang, W. Duan, S. Ahmed, T. E. Mallouk, A. Sen, Nano Today, 2013, 8, 531;

[7] a) M. Wu, Y. Koizumi, H. Nishiyama, I. Tomita, S. Inagi, RSC Adv., 2018, 8, 33331; b) M. Song, M. Cheng, G. Ju, Y. Zhang, F. Shi, Adv. Mater., 2014, 26, 7059. c) L. Zhang, M. Song, M. Xiao, F. Shi, Adv. Funct. Mater., 2016, 26, 851; d) Z. Guo, T. Wang, A. Rawal, J. Hou, Z. Cao, H. Zhang, J. Xu, Z. Gu, V. Chen, K. Liang, Mater. Today 2019, DOI: 10.1016/j.mattod.2019.04.022; e) J. Roche, S. Carrara, J. Sanchez, J. Lannelongue, G. Loget, L. Bouffier, P. Fischer, A. Kuhn, Sci. Rep. 2014, 4, 6705; f) M. Sentic, G. Loget, D. Manojlovic, A. Kuhn, N. Sojic, Angew. Chem. Int. Ed. 2012, 51, 11284; g) G. Loget, A. Kuhn, Lab Chip 2012, 12, 1967;

[8] a) Y. Mai, A. A. Solovev, S. Sánchez, O. G. Schmidt, Chem. Soc. Rev. 2011, 40, 2109; b) H. Wang, M. Pumera, Chem. Rev. 2015, 115, 8704; c) R. María-Hormigos, B. Jurado- Sánchez, A. Escarpa, J. Mater. Chem A. 2016, 28, 8962;

[9] a) G. Loget, D. Zigah, L. Bouffier, N. Sojic, A. Kuhn, Acc. Chem. Res. 2013, 46, 2513; b) S. E. Fosdick, K. N. Knust, K. Scida, R. M. Crooks, Angew. Chem. Int. Ed. 2013, 52, 10538;

[10] a) O. Phuakkong, M. Sentic, H. Li, C. Warakulwit, J. Limtrakul, N. Sojic, A. Kuhn, V. Ravaine, D. Zigah, Langmuir 2016, 32, 12995; b) S. Inagi, Y. Ishiguro, M. Atobe, T. Fuchigami, Angew. Chem. Int. Ed. 2010, 49, 10136; c) L. Zhang, B. Gupta, B. Goudeau, N. Mano, A. Kuhn, J. Am. Chem. Soc. 2018, 140, 15501; d) M. Ongaro, J. Roche, A. Kuhn, P. Ugo, ChemElectroChem. 2014, 1, 2048; e) S. Tiewcharoen, C. Warakulwit, V. Lapeyre, P. Garrigue, L. Fourier, C. Elissalde, S. Bouffière, P. Legros, M. Gayot, J. Limtrakul, A. Kuhn, Angew. Chem. Int. Ed. 2017, 56, 11431;

[11] a) R. W. Jaggers, S. A. F. Bon, Mater. Horiz. 2017, 4, 402; b) J. S. Basuki, F. Qie, X. Mulet, R. Suryadinata, A. V. Vashi, Y. Y. Peng, L. Li, X. Hao, T. Tan, T. C. Hughes, Angew. Chem. Int. Ed. 2017, 56, 966; c) Y. Liang, H. Wang, D. Yao, Y. Chen, Y. Deng, C. Wang, J. Mater. Chem. A. 2017, 5, 18442;

[12] a) J. Hao, W. Yang, Z. Zhang, J. Tang, Nanoscale 2015, 7, 10498; b) R. Sharma, S. T. Chang, O. D. Velev, Langmuir 2012, 28, 10128; c) Y. Liang, Y. Xu, W, Ye, D. Yao, Y. Chen, C. Wang, J. Mater. Chem. A. 2018, 6, 16838; d) H. Wang, X, Gu, C. Wang, ACS Appl. Mater. Interfaces 2016, 8, 9413; e) F. Mou, C. Chen, Q. Zhong, Y. Yin, H. Ma, J. Guan, ACS Appl. Mater. Interfaces 2014, 6, 9897; f) S. V. Nikolov, P. D. Yeh, A. Alexeev, ACS Macro Lett. 2015, 4, 84; g) M. Li, H. Zhang, M. Liu, B. Dong, J. Mater. Chem. C, 2017, 5, 4400, h) M. Medina-Sánchez, V. Magdanz, M. Guix, V. M. Fomin, O. G. Schmidt, Adv. Func. Mater. 2018, 28, 1707228; i) A. Mourran, H. Zhang, R. Vinokur, M. Möller, Adv. Mater. 2017, 29, 1604825;

[13] A. Srinivasan, J. Roche, V. Ravaine, A. Kuhn, Soft Matter 2015, 11, 3958;

[14] a) R. E. Munteanu, L. Stănică, M. Gheorghiu, S. Gáspár, ChemElectroChem., 2019, 6, 1800. b) E. Yariv, J. Fluid Mech. 2010, 655, 105;

[15] a) M. Wu, Y. Koizumi, H. Nishiyama, I. Tomita, S Inagi, RSC Adv., 2018, 8, 33331.

[16] H. Labie, A. Perro, V. Lapeyre, B. Goudeau, B. Catargi, R. Auzély, V. Ravaine, J. Colloid Interface Sci., 2019, 535, 16. 
[18] a) K. S. Huang, M. K. Liu, C. H. Wu, Y. T. Yen, Y. C. Lin, J. Micromech. Microeng. 2007, 17, $1428 ;$ b) R. P. Narayanan, G. Melman, L. J. Letourneau, N. L. Mendelson, A. Melman,

Biomacromolecules 2012, 13, 2465;

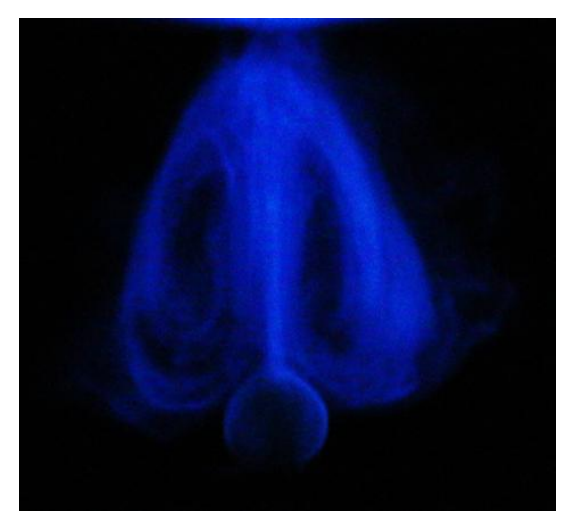

Oscillatory Light-Emitting Biopolymer Based Janus Microswimmers. Non-conductive Janus alginate particles were synthesized via an electrochemical process. Water electrolysis triggers $\mathrm{pH}$ gradients allowing the generation of an asymmetric porosity. These particles are loaded with Prussian Blue precipitates. Prussian Blue catalyzes the formation of oxygen bubbles, which create a buoyancy shift, and enables the chemiluminescence of luminol, turning the particles into light emitting swimmers. 